ein kleiner Ausschnitt aus Hagenbecks Tierpark in Stellingen - die angebliche Wirkung des Friedmannschen Mittels illustriert wird.“ Zur weiteren Aufklärung verweise ich denjenigen Leser, der sich näher informieren will, auf meine Veröffentlichungen' in Nr. 38-39, 43 und 49. Den Artikel von $\mathrm{Nagelschmidt}$ habe ich nur e r w ähnt: Propagandaartikel populärer Zeitschriften werden in der D. m. W. nicht ,besprochen“.

Ich füge hinzu, daß Dr. Nagels $\mathrm{chmidt}$ (nach dem mehrfachen Vorbild von $\mathrm{Fr}$ i edmann und Moses) einen zweiten Propagandaartikel - allerdings ohne die Beweiskraft von Bildern aus Pesterszebet und dem Hagenbeckschen Tierpark - für das Friedmannsche Tuberkulosemittel verfaßt hat, der in mehreren Tageszeitungen erschienen ist. Vielleicht gelingt es $\mathrm{Nag}$ e l s c h m i d t auf diese Weise zu erreichen, daß die deutsche Aerzteschaft (mit geringen Ausnahmen) die Anwendung des Friedmannschen Mittels nicht mehr „sabotiert".

J. S c h wa l be.

\title{
Bemerkung hierzu.
}

Wir hätten diese nach Inhalt und Form zu beanstandende Erklärung nicht a ufgenommen, wenn sie gegen einen anderen Autor gerichtet wäre. Damit der Leser sich selbst ein Urteil bilden kann, wiederhole ich den Satz, der Dr. Nagels $\mathrm{chm}$ id t betrifft, aus meinem Artikel von Nr. 49: "In S c herls $M$ a ga $z$ in hat als Nachfolger des 87jährigen $\mathrm{K}$. K ü s t e r (nicht zu verwechseln mit seinem Bruder, dem Chirurgen, vgl. Nr. 43 S. 1812) F. Na g e l s c h m id t (Berlin), der sich auf dem Gebiete der physikalischen Therapie Verdienste erworben hat, einen Artikel veröffentlicht, in dem nach berühmtem Vorbild F.riedmann und Jenner in Parallele gesetzt wird. in dem von dem „B e w e is Dr. S z a l a i s für die Ausrottbarkeit der Tuberkulose“ die Rede ist und in dem durch 7 Bilder - darunter 